\title{
Systematic review of the nonsurgical management of atlantoaxial rotatory fixation in childhood
}

\author{
Morrakot Sae-Huang, BSc, ${ }^{1}$ Anouk Borg, MD, FRCS, ${ }^{2}$ and Ciaran Scott Hill, PhD, FRCS ${ }^{1,3}$ \\ 'Department of Neurosurgery, The National Hospital for Neurology and Neurosurgery, London; '2Department of Neurosurgery, \\ John Radcliffe Hospital, Oxford; and ${ }^{3}$ University College London Cancer Institute, London, United Kingdom
}

\begin{abstract}
OBJECTIVE Atlantoaxial rotatory fixation (AARF) is an acquired fixed abnormality of C1-2 joint rotation associated with torticollis in childhood. If the condition is left uncorrected, patients are at risk for developing C1-2 fusion with permanent limitation in the cervical range of movement, cosmetic deformity, and impact on quality of life. The management of AARF and the modality of nonsurgical treatment are poorly defined in both primary care and specialized care settings, and the optimal strategy is not clear. This systematic review aims to examine the available evidence to answer key questions relating to the nonsurgical management of AARF.
\end{abstract}

METHODS A systematic review was performed using the following databases: PubMed, MEDLINE, Healthcare Management Information Consortium (HMIC), EMCare, Embase, Cumulative Index to Nursing and Allied Health Literature (CINAHL), British Nursing Index (BNI), and Allied and Complementary Medicine Database (AMED). Search criteria were created and checked independently among the authors. All articles with a radiological diagnosis of AARF and primary outcome data that met the study inclusion criteria were included and analyzed by the authors.

RESULTS Search results did not yield any level I evidence such as a meta-analysis or randomized controlled trial. The initial search yielded 724 articles, 228 of which were screened following application of the core exclusion criteria. A total of 37 studies met the full criteria for inclusion in this review, consisting of 4 prospective studies and 33 retrospective case reviews. No articles directly compared outcomes between modalities of nonsurgical management. Six studies compared the outcome of AARF based on duration of symptoms before initiation of treatment. Comparative analysis of studies was hindered by the wide variety of treatment modalities described and the heterogeneity of outcome data.

CONCLUSIONS The authors did not identify any level I evidence comparing different nonsurgical management approaches for AARF. There were few prospective studies, and most studies were uncontrolled, nonrandomized case series. Favorable outcomes were often reported regardless of treatment methods, with early treatment of AARF tending to yield better outcomes independent of the treatment modality. There is a lack of high-quality data, and further research is required to determine the optimal nonsurgical treatment strategy.

https://thejns.org/doi/abs/10.3171/2020.6.PEDS20396

KEYWORDS systematic review; atlantoaxial rotatory fixation; torticollis; nonsurgical; conservative; spine

A TLANTOAXIAL rotatory fixation (AARF) is a fixedmotion abnormality of $\mathrm{C} 1$ on $\mathrm{C} 2$ that limits rotation of the neck and is associated with painful torticollis. It is typically seen in childhood, with adolescent and adult cases occurring rarely. ${ }^{1-3}$ Clinical diagnosis of AARF is based on fixed rotational deformity of the cervical spine with the head held in a "cock-robin" position with rotation and forward and lateral flexion. ${ }^{1,4,5}$ Without treatment, the atlantoaxial joint is at risk of fusion in the abnormal position, which can cause cosmetic effects, facial asymmetry, functional disability, chronic pain, and compensatory deformity in the subaxial spine. ${ }^{6-8}$

Management strategies for AARF are poorly defined both in the primary care setting and in specialized centers. Incorrect and delayed diagnoses are commonplace. ${ }^{8-10}$ The literature suggests that early closed treatment of AARF can result in excellent outcomes; however, early relapse is well recognized and can necessitate open reduction of

ABBREVIATIONS AARF = atlantoaxial rotatory fixation

SUBMITTED May 13, 2020. ACCEPTED June 18, 2020.

INCLUDE WHEN CITING Published online October 9, 2020; DOI: 10.3171/2020.6.PEDS20396. 
C1-2, usually (though not invariably) with arthrodesis. ${ }^{11-14}$ The use of traction is commonplace, and there are several reports of successful outcomes using traction. However, patient compliance, particularly in the very young, will not infrequently preclude its use in the awake patient. In some, but not all, cases, traction can result in prolonged hospitalization. ${ }^{15-17}$

Early diagnosis, effective initial reduction, and successful retention of cervical positioning are generally considered key to the successful management of AARF in childhood. ${ }^{6,9,12-14,18}$ However, the optimal nonsurgical management strategy has not been clearly defined, and there remain significant variations in treatment strategies for AARF. The relative efficacy of nonsurgical approaches has not been systematically examined. There are two particular points of controversy: what is the most effective way to achieve reduction of the C1-2 deformity, and how best to maintain that reduction in order to reduce the risk of relapse. Therefore, we set out to systematically review the nonsurgical management of AARF and evaluate the relative efficacy of different initial therapeutic approaches.

\section{Methods}

This systematic review was conducted in line with the PRISMA guidelines. ${ }^{19,20}$ The search strategy was run using Boolean operators. The search terms are presented in the Appendix.

The search terms were run on eight databases on May 10, 2020, including PubMed, MEDLINE, Healthcare Management Information Consortium (HMIC), Embase, EMCare, Cumulative Index to Nursing and Allied Health Literature (CINAHL), British Nursing Index (BNI), and Allied and Complementary Medicine Database (AMED), via the National Institute for Health and Care Excellence (NICE) Healthcare Databases Advanced Search (HDAS). Studies mentioned in the articles yielded from the database search that had not been captured by that search were included in the review.

The inclusion criteria were studies in which 1) patients were younger than 18 years old, 2) there was a confirmed radiological diagnosis of AARF, 3) patients had received nonsurgical treatments as the initial treatment, and 4) there were available outcome data that could include range of cervical motion, radiological outcome, rate of recurrence or any complication, morbidity, or quality of life following treatment. Exclusion criteria were applied; these included titles that were not related to AARF, articles without an English-language translation, review articles and articles that only used previously reported data, unpublished posters or conference abstracts, and single case reports (with two patients or fewer).

The authors performed the search strategy independently and reviewed the final papers separately. Any conflicting findings were reviewed by two investigators and consensus was reached. Studies were selected based on detection by the search strategy and fulfillment of the eligibility criteria. Types of studies eligible for selection included meta-analyses, randomized controlled trials, observational studies, and retrospective case reviews or case series. All duplicates were removed first. Article titles and abstracts were then reviewed in the screening step, followed by full-text article assessment for eligibility. The full text of each article was reviewed for relevance. Data items extracted from each article included author names, article title, year of publication, study type, and PICO (population, intervention, comparison, outcome) framework. The PICO elements specifically included number of patients, average age, type of nonsurgical treatments received, any comparison made between patient groups, and primary outcome measure from each article. Any bias or limitation at both the study and outcome level of each article was noted during the data collection process. Primary summary measures were the method of nonsurgical treatment performed at the reduction stage and stabilization stage and the treatment outcomes.

\section{Results}

Search results are presented using the PRISMA 2009 flow diagram in Fig. 1. After applying our inclusion criteria, 37 studies were included in our systematic review. There were 33 retrospective case series and 4 prospective studies. These are summarized in Tables 1 and 2, respectively.

Our search did not yield any level I evidence such as a meta-analysis or randomized controlled trial. None of the 37 papers compared outcomes between two different types of nonsurgical treatments. A wide variety of nonsurgical treatment methods were described in the literature, with different combinations of methods used in the reduction stage and retention stage. A summary of nonsurgical treatment methods applied at each stage is presented in Table 3.

\section{Discussion}

A patient with AARF passes through a number of stages during their treatment journey. These include initial clinical and radiographic diagnosis, then a decision to attempt nonsurgical management or operative reduction/fixation. If the nonsurgical treatment route is selected, then decisions must be made regarding the method of reduction and subsequent retention plus the duration of initial immobilization. If there is a failure of reduction or a subsequent recurrence of AARF, then either second-line (or third-line) nonoperative intervention may be considered or a definitive operation performed. Making the best decisions to maximize successful patient outcomes at each of these time points is challenging. Such decisions should be guided by evidence when possible. In this systematic review, we assessed the evidence for nonsurgical management decisions at critical points in the management of AARF and evaluated the relative efficacy of different initial therapeutic approaches.

Key steps in the nonsurgical treatment pathway in relation to evidence obtained from this systematic review is outlined below.

\section{Selection of Patients for Nonsurgical Reduction}

This study specifically concerns evidence relating to nonsurgical treatments for AARF. Surgical management 


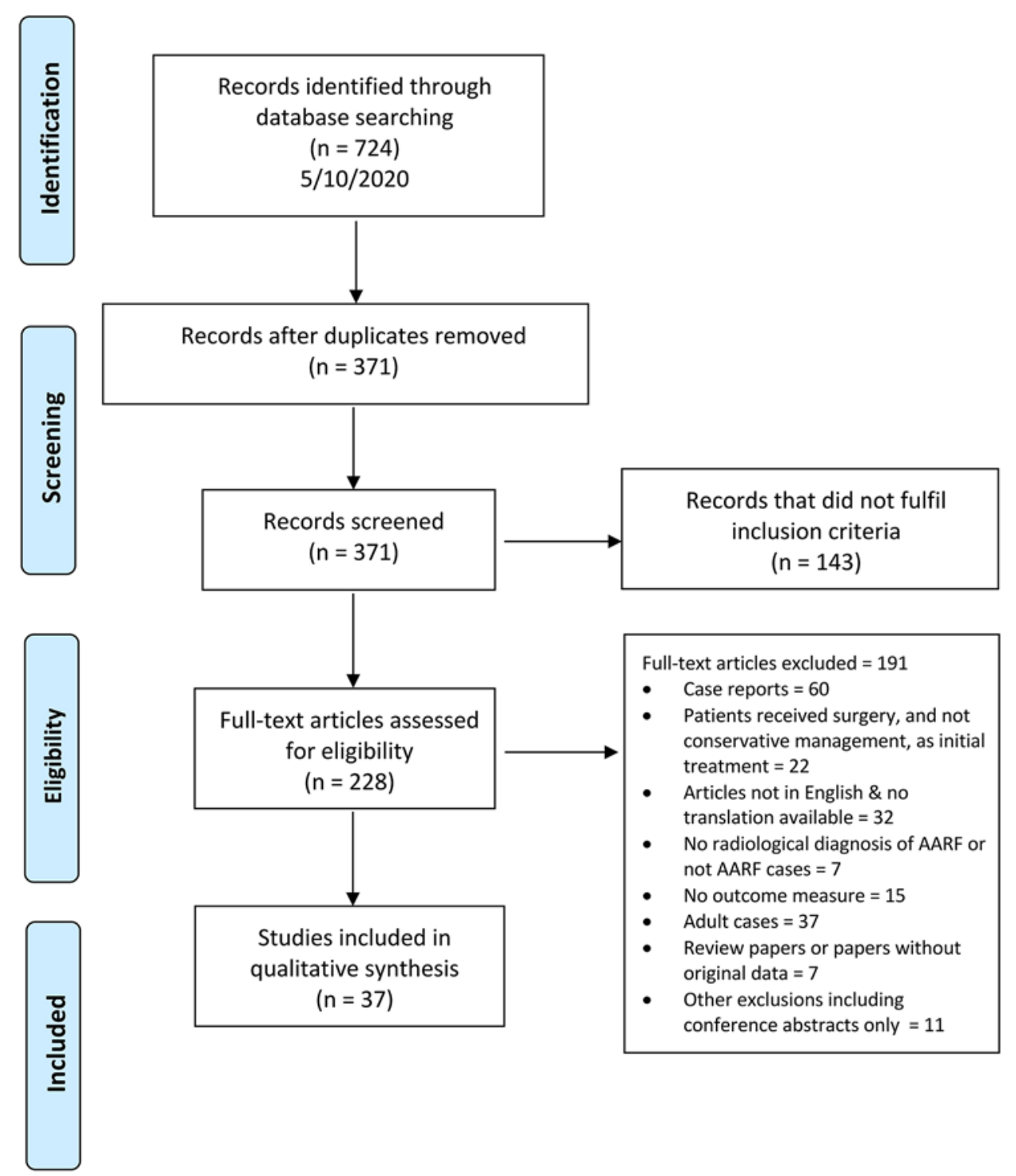

FIG. 1. PRISMA 2009 flow diagram. This systematic review was performed in accordance with the PRISMA 2009 guidelines and includes details on record identification, screening, eligibility and exclusions, and final inclusions. Figure is available in color online only.

was indicated after a failure to reduce, after frequent recurrences following a trial of conservative treatment, or in the cases in which complications of conservative treatment arose. Controversy remains regarding whether neurological involvement should be an indication for surgery without a preceding trial of conservative management. While Ortiz and colleagues opted for fusion surgery in a case with hemiparesis, Missori et al. described successful conservative therapy in a case presenting with transient visual loss due to vertebrobasilar compression. ${ }^{26,30}$ There seems to be a general consensus toward conservative treatment in acute cases without neurological deficit and fusion in chronic cases, but these are not universal practices.7,13,28,33,52 The duration of symptoms that define an "acute" or a "chronic" case varies among studies and makes direct comparisons challenging.

\section{Method of Initial Nonsurgical Reduction}

In many instances, AARF is managed with simple nonsurgical measures such as analgesia, muscle relaxants, and collars without manipulation under anesthesia (MUA)..$^{24,53}$ However, where torticollis does not resolve promptly, escalation of treatment to include either nonsurgical reduction or surgical intervention is generally considered essential to avoid a poor long-term outcome. There was no consensus on the method of reduction among the different studies. There was also no clear explanation for choosing one method of reduction over the next, and neither was there any study with a direct comparative analysis. The described methods of initial nonsurgical reduction included no intervention (spontaneous reduction), antiinflammatories with or without muscle relaxants with or without analgesia with or without physiotherapy, manual 
TABLE 1. Case series included in the systematic review

\begin{tabular}{|c|c|c|c|c|c|c|c|c|}
\hline $\begin{array}{l}\text { Authors } \\
\& \text { Year }\end{array}$ & $\begin{array}{l}\text { Study } \\
\text { Type }\end{array}$ & $\begin{array}{l}\text { Level of } \\
\text { Evidence }\end{array}$ & $\begin{array}{c}\text { No. of } \\
\text { Patients }\end{array}$ & $\begin{array}{l}\text { Average } \\
\text { Patient Age in } \\
\text { Years (range) }\end{array}$ & Intervention & FU & Comparison & $\begin{array}{c}\text { Outcome } \\
\text { Described/Notes }\end{array}$ \\
\hline $\begin{array}{l}\text { Patel } \\
\text { et al., } \\
2020^{21}\end{array}$ & $\begin{array}{l}\text { Case } \\
\text { series }\end{array}$ & IV & 3 & $7(6-9)$ & $\begin{array}{l}\text { Reduction: cervical traction, aver- } \\
\text { age } 13 \text { days, } n=3 \text {; retention: halo, } \\
\text { average } 47 \text { days, } n=3\end{array}$ & $\begin{array}{l}\text { Range } 5 \\
\text { mos-10 } \\
\text { yrs }\end{array}$ & $\begin{array}{l}\text { No compari- } \\
\text { son }\end{array}$ & $\begin{array}{l}\text { No recurrence, no signs of } \\
\text { subluxation on CT, no clinical } \\
\text { symptoms, } n=3 \text {; full ROM, } \\
n=1\end{array}$ \\
\hline $\begin{array}{l}\text { laccarino } \\
\text { et al., } \\
2019^{22}\end{array}$ & $\begin{array}{l}\text { Case } \\
\text { series }\end{array}$ & IV & 5 & $7.4(5-12)$ & $\begin{array}{l}\text { Reduction: antibiotic \& analgesia, } \\
n=2 \text {; retention: cervical collar, } \\
\text { average } 6 \text { wks, } n=2 \text {; reduction: } \\
\text { Gardner-Wells traction, } 4 \text { days, } \\
\text { after failure to reduce by MUA, } n \\
=1 \text {; retention: SOMI, } 12 \text { wks, } n=1\end{array}$ & $\begin{array}{l}\text { Range } \\
4 \text { wks-2 } \\
\text { mos }\end{array}$ & $\begin{array}{l}\text { No compari- } \\
\text { son }\end{array}$ & $\begin{array}{l}\text { Symptoms \& subluxation } \\
\text { resolved on MRI FU, } n=3 ; \\
\text { near-normal alignment on CT } \\
\text { FU in patient w/ Gardner-Wells } \\
\text { traction, } n=1\end{array}$ \\
\hline $\begin{array}{l}\text { Ozalp } \\
\text { et al., } \\
2019^{23}\end{array}$ & $\begin{array}{l}\text { Case } \\
\text { series }\end{array}$ & IV & 16 & $9(3-14)$ & $\begin{array}{l}\text { Reduction: MUA followed by } \\
\text { antibiotics \& NSAIDs, } n=16 \text {; } \\
\text { retention: Philadelphia collar, } 2-4 \\
\text { wks, } n=15 ; \text { retention: soft neck } \\
\text { collar, } 2-4 \text { wks, } n=1\end{array}$ & NA & $\begin{array}{l}\text { No compari- } \\
\text { son }\end{array}$ & $\begin{array}{l}\text { Successfully reduced, } n=15 \text {; } \\
\text { reduction failed, } n=1\end{array}$ \\
\hline $\begin{array}{l}\text { Powell } \\
\text { et al., } \\
2017^{24}\end{array}$ & $\begin{array}{l}\text { Sec- } \\
\text { ondary } \\
\text { analysis } \\
\text { of case- } \\
\text { control } \\
\text { study }\end{array}$ & IV & $55^{*}$ & 7.7 & $\begin{array}{l}\text { No intervention: } n=6 \text {; cervical } \\
\text { collar alone, } n=24 \text {; halo ring, } n= \\
9 ; \text { halo traction, } n=14 ; \text { surgery: } \\
\text { internal fixation, } n=2\end{array}$ & NA & $\begin{array}{l}\text { Injury } \\
\text { mechanism } \\
\& \text { type of } \\
\text { treatment }\end{array}$ & Persistent deficit, $n=6$ \\
\hline $\begin{array}{l}\text { Mifsud } \\
\text { et al., } \\
2016^{18}\end{array}$ & $\begin{array}{l}\text { Case } \\
\text { series }\end{array}$ & IV & 12 & $7.3(1.5-13.4)$ & $\begin{array}{l}\text { Reduction: MUA followed by halo } \\
\text { traction, average } 3.6 \text { wks, } n=11 \text {; } \\
\text { fusion surgery, } n=1 \text {; retention: } \\
\text { halo vest jacket, } n=8 \text {; Minerva } \\
\text { jacket, } n=4 \text {; average } 6.9 \text { wks in } \\
\text { retention }\end{array}$ & $\begin{array}{l}\text { Range } \\
24-100 \\
\text { wks }\end{array}$ & $\begin{array}{l}\text { No compari- } \\
\text { son }\end{array}$ & $\begin{array}{l}\text { Symptom free, full ROM, } n \\
=10 ; \text { recurrence requiring } \\
\text { fusion, } n=1\end{array}$ \\
\hline $\begin{array}{l}\text { Ocak \& } \\
\text { Ocak, } \\
2016^{25}\end{array}$ & $\begin{array}{l}\text { Case } \\
\text { series }\end{array}$ & IV & 7 & $7.8(5-13)$ & $\begin{array}{l}\text { Reduction: MUA, } n=7 \text {; retention: } \\
\text { Philadelphia collar, } 6 \text { wks, } n=7\end{array}$ & NA & $\begin{array}{l}\text { No compari- } \\
\text { son }\end{array}$ & $\begin{array}{l}\text { No pain, no neurological } \\
\text { abnormality, } n=7\end{array}$ \\
\hline $\begin{array}{l}\text { Missori } \\
\text { et al., } \\
2014^{26}\end{array}$ & $\begin{array}{l}\text { Case } \\
\text { series }\end{array}$ & IV & 14 & $5.5(3-9)$ & $\begin{array}{l}\text { Reduction: spontaneous reduction } \\
\text { in Schanz or Philadelphia collar } \\
\text { w/ bed rest, average } 8 \mathrm{hrs}, \mathrm{n}=14 \text {; } \\
\text { retention: Schanz or Philadelphia } \\
\text { collar, } 14 \text { days, } n=14\end{array}$ & $\begin{array}{l}\text { Average } \\
3.3 \text { yrs }\end{array}$ & $\begin{array}{l}\text { No compari- } \\
\text { son }\end{array}$ & $\begin{array}{l}\text { Successfully reduced, no } \\
\text { recurrence, no abnormality in } \\
\text { ADLs, } n=14\end{array}$ \\
\hline $\begin{array}{l}\text { Akbay } \\
\text { et al., } \\
2014^{27}\end{array}$ & $\begin{array}{l}\text { Case } \\
\text { series }\end{array}$ & IV & 12 & $7.17(4-12)$ & $\begin{array}{l}\text { Reduction: MUA, } n=12 \text {; retention: } \\
\text { rigid or semi-rigid cervical ortho- } \\
\text { sis, } 4 \text { wks, \& muscle relaxants, } 1 \\
\text { wk, } n=12\end{array}$ & $\begin{array}{l}\text { Range } \\
6-24 \\
\text { mos }\end{array}$ & $\begin{array}{l}\text { No compari- } \\
\text { son }\end{array}$ & $\begin{array}{l}\text { Successfully reduced, } n=12 \text {; } \\
\text { no recurrence, } n=11 ; \text { recur- } \\
\text { rence requiring } 2 \text { nd closed } \\
\text { reduction maneuver, } n=1\end{array}$ \\
\hline $\begin{array}{l}\text { Glotz- } \\
\text { becker } \\
\text { et al., } \\
2014^{28}\end{array}$ & $\begin{array}{l}\text { Case } \\
\text { series }\end{array}$ & IV & 14 & $7.2(1.5-12)$ & $\begin{array}{l}\text { Reduction: halter traction or } \\
\text { noninvasive halo or halo traction, } \\
n=11 \text {; retention: halo vest, } n= \\
N A \text {; reduction: } C 1-2 \text { fusion after } \\
\text { reduction, } n=3 \text {; retention: NA }\end{array}$ & $\begin{array}{l}\text { Average } \\
10.3 \\
\text { mos } \\
\text { (range } \\
3.5-42 \\
\text { mos) }\end{array}$ & $\begin{array}{l}\text { Age \& } \\
\text { symptom } \\
\text { duration }\end{array}$ & $\begin{array}{l}\text { Successful conservative } \\
\text { treatment, } n=8 \text {; no pain, } n= \\
6 \text {; minimal occasional pain, } n= \\
1 ; \text { full ROM, } n=5 \text {; loss of rota- } \\
\text { tion, } n=2 ; \text { conservative treat- } \\
\text { ment failed, requiring fusion, } \\
n=3 \text {; limited ROM following } \\
\text { fusion, } n=3 ; 75 \% \text { success } \\
\text { rate for those } w / \text { chronic AARF }\end{array}$ \\
\hline $\begin{array}{l}\text { Tauchi } \\
\text { et al., } \\
2013^{29}\end{array}$ & $\begin{array}{l}\text { Case } \\
\text { series }\end{array}$ & IV & 11 & $11(6-17)$ & $\begin{array}{l}\text { All had chronic AARF that did } \\
\text { not respond to at least } 3 \text { mos of } \\
\text { nonsurgical treatment w/ neck col- } \\
\text { lar, neck traction, Minerva jacket, } \\
\text { or halo vest; thus, all had fusion } \\
\text { surgery }\end{array}$ & $\begin{array}{l}2 \text { yrs } 2 \\
\text { mos-9 } \\
\text { yrs } 1 \text { mo }\end{array}$ & $\begin{array}{l}\text { No compari- } \\
\text { son }\end{array}$ & $\begin{array}{l}\text { Cervical ROM decreased in all } \\
\text { patients after surgery }\end{array}$ \\
\hline
\end{tabular}


» CONTINUED FROM PAGE 4

TABLE 1. Case series included in the systematic review

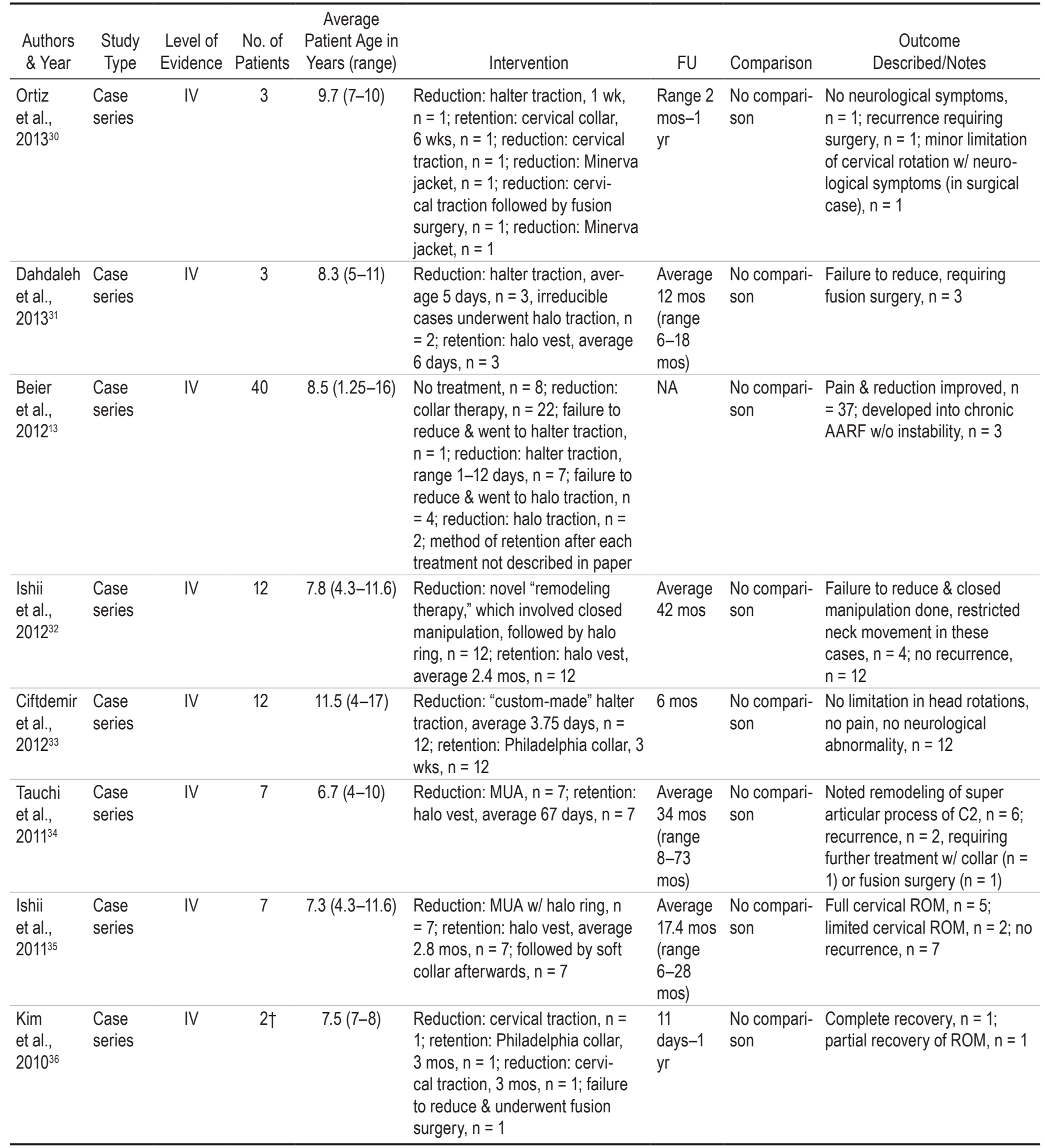


» CONTINUED FROM PAGE 5

TABLE 1. Case series included in the systematic review

\begin{tabular}{|c|c|c|c|c|c|c|c|c|}
\hline $\begin{array}{l}\text { Authors } \\
\text { \& Year }\end{array}$ & $\begin{array}{l}\text { Study } \\
\text { Type }\end{array}$ & $\begin{array}{l}\text { Level of } \\
\text { Evidence }\end{array}$ & $\begin{array}{l}\text { No. of } \\
\text { Patients }\end{array}$ & $\begin{array}{l}\text { Average } \\
\text { Patient Age in } \\
\text { Years (range) }\end{array}$ & Intervention & FU & Comparison & $\begin{array}{c}\text { Outcome } \\
\text { Described/Notes }\end{array}$ \\
\hline $\begin{array}{l}\text { Wang } \\
\text { et al., } \\
2008^{37}\end{array}$ & $\begin{array}{l}\text { Case } \\
\text { series }\end{array}$ & IV & 3 & $9(7-10)$ & $\begin{array}{l}\text { Reduction: soft neck traction, } 6 \\
\text { wks, } n=1 ; \text { retention: neck collar, } n \\
=1 ; \text { reduction: soft neck traction, } \\
1 \text { wk, } n=1 ; \text { retention: halo vest, } n \\
=1 ; \text { reduction: soft neck traction, } \\
1 \text { mo, } n=1 ; \text { failure to reduce \& } \\
\text { underwent fusion surgery, } n=1\end{array}$ & $\begin{array}{l}\text { Range } \\
6 \text { wks-3 } \\
\text { mos }\end{array}$ & $\begin{array}{l}\text { No compari- } \\
\text { son }\end{array}$ & $\begin{array}{l}\text { Complete recovery, } n=1 \text {; neck } \\
\text { in neutral position, } n=1 \text {; recur- } \\
\text { rence after fusion surgery, } \\
n=1\end{array}$ \\
\hline $\begin{array}{l}\text { Ishii } \\
\text { et al., } \\
2006^{38}\end{array}$ & $\begin{array}{l}\text { Case } \\
\text { series }\end{array}$ & IV & 24 & $7.9(4-14)$ & $\begin{array}{l}\text { Acute cases: reduction, skull } \\
\text { traction, } n=7 \text {; retention, rigid } \\
\text { orthosis, average } 2 \text { wks, } n=7 \text {; } \\
\text { reduction, NA, } n=8 \text {; retention, } \\
\text { Miami J or Philadelphia collar, } n= \\
8 \text {; chronic cases: reduction, skull } \\
\text { traction, } n=9 \text {; retention, rigid } \\
\text { orthosis, } n=4\end{array}$ & $\begin{array}{l}\text { Average } \\
3.7 \text { yrs } \\
\text { (range } 5 \\
\text { wks-12 } \\
\text { yrs) }\end{array}$ & $\begin{array}{l}\text { Symptom } \\
\text { duration }\end{array}$ & $\begin{array}{l}\text { No recurrence in acute cases } \\
\& \text { all neurologically intact } \\
\text { w/ full ROM, } n=15 \text {; recur- } \\
\text { rences in chronic cases, } n=4 ; \\
\text { surgery for cases } w / \text { failure to } \\
\text { reduce among chronic cases, } \\
n=5\end{array}$ \\
\hline $\begin{array}{l}\text { Chiappa- } \\
\text { rini et al., } \\
2005^{39}\end{array}$ & $\begin{array}{l}\text { Case } \\
\text { series }\end{array}$ & IV & 4 & $9.5(4-13)$ & $\begin{array}{l}\text { Reduction: Gardner tong traction, } \\
n=3 \text {; retention: soft collar, } 4 \text { wks, } \\
n=2 \text {; no treatment, } n=1\end{array}$ & $\begin{array}{l}\text { Range } \\
3-20 \\
\text { mos }\end{array}$ & $\begin{array}{l}\text { No compari- } \\
\text { son }\end{array}$ & $\begin{array}{l}\text { Spontaneously resolved } w / 0 \\
\text { treatment, } n=1 \text {; completely } \\
\text { recovered, } n=2 \text {; mild restric- } \\
\text { tion of head rotation, } n=1\end{array}$ \\
\hline $\begin{array}{l}\text { Rahimi } \\
\text { et al., } \\
2003^{40}\end{array}$ & $\begin{array}{l}\text { Case } \\
\text { series }\end{array}$ & IV & 6 & $7.5(1.8-14.6) \ddagger$ & $\begin{array}{l}\text { Reduction: cervical traction, } \\
48-72 \text { hrs, } n=3 \text {; reduction: NA, } \\
\text { "reduced spontaneously w/ use } \\
\text { of rigid cervical collar," } n=2 ; \\
\text { retention: cervical collar, } n=5 ; \\
\text { reduction: halo traction, } 3 \text { mos, } n \\
=1 \text {; retention: NA }\end{array}$ & NA & $\begin{array}{l}\text { No compari- } \\
\text { son }\end{array}$ & $\begin{array}{l}\text { Recurrence, } n=1 \text {; full neuro- } \\
\text { logical recovery, } n=1\end{array}$ \\
\hline $\begin{array}{l}\text { Fernán- } \\
\text { dez } \\
\text { Cornejo } \\
\text { et al., } \\
2003^{41}\end{array}$ & $\begin{array}{l}\text { Case } \\
\text { series }\end{array}$ & IV & 4 & $7.5(4-13)$ & $\begin{array}{l}\text { Reduction: conservative } w / \text { anti- } \\
\text { inflammatory \& physiotherapy by } \\
\text { gentle head traction, } n=4 \text {; reten- } \\
\text { tion: hard collar, } n=1 \text {; retention: } \\
\text { SOMl brace, } n=2\end{array}$ & $\begin{array}{l}\text { Range } 1 \\
\text { wks-4.5 } \\
\text { yrs }\end{array}$ & $\begin{array}{l}\text { No compari- } \\
\text { son }\end{array}$ & $\begin{array}{l}\text { Full ROM, } n=3 \text {; full recov- } \\
\text { ery according to } C T, n=3 \text {; } \\
\text { restricted ROM, } n=1\end{array}$ \\
\hline $\begin{array}{l}\text { Lee } \\
\text { et al., } \\
2002^{42}\end{array}$ & $\begin{array}{l}\text { Case } \\
\text { series }\end{array}$ & IV & 6 & $9(7-12)$ & $\begin{array}{l}\text { Reduction: halter traction, } n=6 \text {; } \\
\text { retention: halo jacket, } n=3\end{array}$ & $\begin{array}{l}\text { At least } \\
18 \text { mos }\end{array}$ & $\begin{array}{l}\text { No compari- } \\
\text { son }\end{array}$ & $\begin{array}{l}\text { Recurrence requiring fusion } \\
\text { surgery, } n=3\end{array}$ \\
\hline $\begin{array}{l}\text { McGuire } \\
\text { et al., } \\
2002^{43}\end{array}$ & $\begin{array}{l}\text { Case } \\
\text { series }\end{array}$ & IV & 50 & 8.2 & $\begin{array}{l}\text { No treatment, } n=4 \text {; NSAIDS, } \\
\text { antibiotics, physical therapy, \&/ } \\
\text { or soft collar, } n=16 \text {; hard collar, } \\
n=9 ; \text { halter traction, } n=12 ; \text { cer- } \\
\text { vicothoracic orthosis, } n=3 \text {; halo } \\
\text { traction, } n=2 ; \text { not reported, } n=4\end{array}$ & NA & $\begin{array}{l}\text { No compari- } \\
\text { son }\end{array}$ & $\begin{array}{l}\text { Successfully treated, } n=50 ; \\
\text { no significant differences in } \\
\text { age at onset among differ- } \\
\text { ent stages \& no significant } \\
\text { difference btwn groups in } \\
\text { terms of duration of symptoms; } \\
\text { significant trend toward higher } \\
\text { level of treatment intervention } \\
\text { w/ more severe stage }\end{array}$ \\
\hline $\begin{array}{l}\text { Martinez- } \\
\text { Lage } \\
\text { et al., } \\
2001^{44}\end{array}$ & $\begin{array}{l}\text { Case } \\
\text { series }\end{array}$ & IV & 4 & $8.2(5-13)$ & $\begin{array}{l}\text { Reduction: conservative w/ ben- } \\
\text { zodiazepines, anti-inflammatories, } \\
\& \text { physiotherapy including head } \\
\text { traction, } 2-6 \text { wks, } n=3 \text {; retention: } \\
\text { cervical collar, } 4-6 \text { wks, } n=3 \text {; } \\
\text { reduction: conservative w/ medi- } \\
\text { cal treatment; retention: Minerva } \\
\text { vest, } n=1\end{array}$ & $\begin{array}{l}\text { Range } \\
1-5 \text { yrs }\end{array}$ & $\begin{array}{l}\text { No compari- } \\
\text { son }\end{array}$ & $\begin{array}{l}\text { Full ROM, } n=3 \text {; failure to re- } \\
\text { duce, requiring fusion surgery, } \\
n=1\end{array}$ \\
\hline
\end{tabular}


» CONTINUED FROM PAGE 6

TABLE 1. Case series included in the systematic review

\begin{tabular}{|c|c|c|c|c|c|c|c|c|}
\hline $\begin{array}{l}\text { Authors } \\
\& \text { Year }\end{array}$ & $\begin{array}{l}\text { Study } \\
\text { Type }\end{array}$ & $\begin{array}{l}\text { Level of } \\
\text { Evidence }\end{array}$ & $\begin{array}{c}\text { No. of } \\
\text { Patients }\end{array}$ & $\begin{array}{l}\text { Average } \\
\text { Patient Age in } \\
\text { Years (range) }\end{array}$ & Intervention & FU & Comparison & $\begin{array}{c}\text { Outcome } \\
\text { Described/Notes }\end{array}$ \\
\hline $\begin{array}{l}\text { Mihara } \\
\text { et al., } \\
2001^{16}\end{array}$ & $\begin{array}{l}\text { Case } \\
\text { series }\end{array}$ & IV & 35 & $6.5(2-12)$ & $\begin{array}{l}\text { Reduction: halter traction, } \mathrm{n}=35 \\
\text { retention: soft collar, } 1 \mathrm{mo}, \mathrm{n}=35\end{array}$ & $\begin{array}{l}\text { Average } \\
3.4 \text { yrs } \\
\text { (range } \\
1.4-5.8 \\
\text { yrs) }\end{array}$ & $\begin{array}{l}\text { Age \& } \\
\text { symptom } \\
\text { duration }\end{array}$ & $\begin{array}{l}\text { Recurrence, } n=9 \text {, further } \\
\text { treated w/ soft collar ( } n=5 \text { ) or } \\
\text { continuous cervical traction } \\
(n=4) \text {; recurrence highest in } \\
6 \text { - to 9-yr-old patients but no } \\
\text { significant difference btwn } \\
\text { age subgroups; recurrence } \\
\text { developed in all patients ( } n= \\
\text { 4) whose symptom duration } \\
>1 \text { wk, \& their recurrence rate } \\
\text { significantly higher than others }\end{array}$ \\
\hline $\begin{array}{l}\text { Holcomb } \\
\text { et al., } \\
2001^{45}\end{array}$ & $\begin{array}{l}\text { Case } \\
\text { series }\end{array}$ & IV & 4 & $7.8(7-9)$ & $\begin{array}{l}\text { Reduction: halo traction, aver- } \\
\text { age } 3.6 \text { days, } n=3 \text {; retention: } \\
\text { halo vest, average } 8 \text { wks, } n=3 \text {, } \\
\text { followed by Philadelphia collar, } 2 \\
\text { wks, } n=3 \text {, then soft collar, } n=1 \text {; } \\
\text { reduction: MUA, } n=1 \text {; retention: } \\
\text { Miami J collar, } 12 \text { wks, followed } \\
\text { by Philadelphia collar, } 2 \text { wks, then } \\
\text { soft collar, } n=1\end{array}$ & $\begin{array}{l}\text { Range } \\
2 \text { wks-9 } \\
\text { mos }\end{array}$ & $\begin{array}{l}\text { No compari- } \\
\text { son }\end{array}$ & $\begin{array}{l}\text { Normal bony alignment shown } \\
\text { by spine films, } n=1 \text {; activity } \\
\text { restriction lifted immediately, } \\
n=1 \text {; activity restriction lifted } \\
\text { after } 5 \text { mos, } n=1 \text {; activity } \\
\text { restriction lifted after } 9 \text { mos, } n \\
=1 \text {; outcome NA, } n=1\end{array}$ \\
\hline $\begin{array}{l}\text { Herzka } \\
\text { et al., } \\
2000^{46}\end{array}$ & $\begin{array}{l}\text { Case } \\
\text { series }\end{array}$ & IV & $2 \S$ & $11.3(9.5-13)$ & $\begin{array}{l}\text { Reduction: tong traction failed to } \\
\text { reduce, thus underwent MUA, } n= \\
1 \text {; retention: } 2 \text {-post cervical collar, } \\
n=1 ; \text { reduction: halter traction, } 20 \\
\text { days, } n=1 \text {; retention: collar, } n=1\end{array}$ & NA & NA & $\begin{array}{l}\text { Subluxation failed to reduce } \\
\text { after collar, thus referred for } \\
\text { fusion surgery, } n=1 \text {; problem } \\
\text { did not recur in another case, } \\
n=1\end{array}$ \\
\hline $\begin{array}{l}\text { Schwarz, } \\
1998^{6}\end{array}$ & $\begin{array}{l}\text { Case } \\
\text { series }\end{array}$ & IV & 4 & $5.8(2-13)$ & $\begin{array}{l}\text { Reduction: MUA, } n=3 \text {; Minerva } \\
\text { cast, } n=2 \text {; reduction: surgery, } \\
n=1\end{array}$ & $\begin{array}{l}\text { Range } 7 \\
\text { mos-2 } \\
\text { yrs }\end{array}$ & $\begin{array}{l}\text { No compari- } \\
\text { son }\end{array}$ & $\begin{array}{l}\text { Complete clinical \& radio- } \\
\text { graphic recovery, } n=1 ; \text { pain } \\
\text { free \& limited } R O M, n=1 ; \\
\text { failed to reduce, } n=1\end{array}$ \\
\hline $\begin{array}{l}\text { Johnson } \\
\text { \& Fer- } \\
\text { gusson, } \\
1986^{49}\end{array}$ & $\begin{array}{l}\text { Case } \\
\text { series }\end{array}$ & IV & $2 \pi$ & $11.5(11-12)$ & $\begin{array}{l}\text { Reduction: halter traction, } 1 \text { wk, } \\
n=2 \text {; retention: soft collar, } 6 \text { wks, } \\
n=2\end{array}$ & $3 \mathrm{mos}$ & $\begin{array}{l}\text { No compari- } \\
\text { son }\end{array}$ & $\begin{array}{l}\text { Clinically normal, } n=2 \text {; radio- } \\
\text { graphically normal, } n=2\end{array}$ \\
\hline
\end{tabular}


$\mathrm{ADL}=$ activity of daily living; FU = follow-up; MUA = manipulation under anesthesia; NA = not available; NSAID = nonsteroidal anti-inflammatory drug; ROM = range of motion; SOMI = sternal occipital mandibular immobilizer.

${ }^{*}$ Fifty-five patients with AARF; total of 540 children with cervical spine injuries.

$\dagger$ An adult case was excluded.

$\ddagger$ Age range from 23 patients with atlantoaxial injuries; not specific to the 6 patients with AARF.

$\S$ Case 3 was an adult case and so was not included.

I Case 3 was not AARF and was thus excluded.

reduction under anesthesia, halter traction, and halo or Gardner-Wells traction. In addition to the above, two studies described novel therapy designed for their respective study groups, which included "custom-made" occipitomental traction and a form of modified halter traction. ${ }^{32,33}$ Among the included papers, halter traction was the most frequently described intervention for reduction, at 11/37 studies $(30 \%)$. Retention alone by different types of collars or braces, without any described reduction by traction or manipulation, was the most commonly described overall treatment modality, at 13/37 studies (35\%).

\section{Method of Maintaining Reduced Position}

A wide variety of methods of retention after successful reduction were described in the literature, again without a consistent rationale for why each type was chosen. The

TABLE 2. Prospective studies included in the systematic review

\begin{tabular}{|c|c|c|c|c|c|c|c|c|}
\hline $\begin{array}{l}\text { Authors } \\
\& \text { Year }\end{array}$ & $\begin{array}{l}\text { Study } \\
\text { Type }\end{array}$ & $\begin{array}{l}\text { Level of } \\
\text { Evidence }\end{array}$ & $\begin{array}{c}\text { No. of } \\
\text { Patients }\end{array}$ & $\begin{array}{l}\text { Average } \\
\text { Patient Age in } \\
\text { Years (range) }\end{array}$ & Intervention & FU & Comparison & $\begin{array}{c}\text { Outcome } \\
\text { Described/Notes }\end{array}$ \\
\hline $\begin{array}{l}\text { Landi } \\
\text { et al., } \\
2012^{50}\end{array}$ & $\begin{array}{l}\text { Prospective } \\
\text { nonran- } \\
\text { domized } \\
\text { study }\end{array}$ & III & 9 & $5.7(4-8)$ & $\begin{array}{l}\text { Reduction: Schanz collar, } \\
n=9 \text {; retention: Philadel- } \\
\text { phia collar, } n=9\end{array}$ & $1 \mathrm{yr}$ & None & $\begin{array}{l}\text { Successful reduction per MRI }(n=9) \text {; } \\
\text { inflammatory edematous phenomena at } \\
3 \text { mos per MRI }(n=4) \text {; symptom free at } \\
1 \text { yr }(n=9)\end{array}$ \\
\hline $\begin{array}{l}\text { Pang, } \\
2010^{12}\end{array}$ & $\begin{array}{l}\text { Prospective } \\
\text { nonran- } \\
\text { domized } \\
\text { study }\end{array}$ & III & 35 & $8.4(1.5-14.5)$ & $\begin{array}{l}\text { Acute \& subacute cases: } \\
\text { reduction, halter traction, } \\
n=19 ; \text { retention, Guilford } \\
\text { brace, } 3 \text { mos, } n=19 ; \\
\text { chronic cases: reduction, } \\
\text { skull traction by calipers } \\
\text { or halo rings, } n=10 ; \\
\text { retention, halo vest, } n=10\end{array}$ & NA & $\begin{array}{l}\text { Symptom } \\
\text { duration \& } \\
\text { AARF type }\end{array}$ & $\begin{array}{l}\text { No acute patients required halo fixation } \\
\text { or surgical fusion, whereas subacute } \\
\text { patients had longer duration of treatment, } \\
\& \text { all required halo fixation, w/ } 1 \text { needing } \\
\text { fusion; none resumed normal } C 1-2 \\
\text { dynamics; in chronic patients, reduction } \\
\text { never achieved; } n=5 \text { required fusion; } \\
\text { only } n=1 \text { resumed normal C } 1-2 \text { dynam- } \\
\text { ics after multiple courses of traction; } \\
\text { chronic group had longer traction dura- } \\
\text { tion, more recurrences, \& high nonreduc- } \\
\text { ible rate, compared to acute \& subacute } \\
\text { groups; subacute group outcome closer } \\
\text { to chronic AARF than acute AARF }\end{array}$ \\
\hline $\begin{array}{l}\text { Pang } \\
\& \mathrm{Li} \\
2005^{7}\end{array}$ & $\begin{array}{l}\text { Prospective } \\
\text { nonran- } \\
\text { domized } \\
\text { study }\end{array}$ & III & $29^{*}$ & $7.9(1.1-12.5)$ & $\begin{array}{l}\text { Acute \& subacute cases: } \\
\text { reduction, halter traction, } \\
n=19 ; \text { retention, Guilford } \\
\text { brace, } 3 \text { mos, } n=19 ; \\
\text { chronic cases: reduction, } \\
\text { skull traction by calipers } \\
\text { or halo rings, } n=10 ; \\
\text { retention, halo vest, } n=10\end{array}$ & NA & $\begin{array}{l}\text { Symptom } \\
\text { duration \& } \\
\text { AARF type }\end{array}$ & $\begin{array}{l}\text { Successful reduction in all acute patients } \\
\& \text { some subacute } \& \text { chronic patients, } n \\
=22 \text {; failure to reduce in some subacute } \\
\& \text { chronic patients, } n=6 \text {; fusion needed, } \\
n=6 \text {, none in acute group; normal final } \\
\text { C1-2 dynamics in acute }(n=12) \text {, sub- } \\
\text { acute }(n=2), \& \text { chronic }(n=2) \text { groups }\end{array}$ \\
\hline $\begin{array}{l}\text { Nich- } \\
\text { olson } \\
\text { et al., } \\
1999^{51}\end{array}$ & $\begin{array}{l}\text { Prospective } \\
\text { nonran- } \\
\text { domized } \\
\text { study }\end{array}$ & III & $13 \dagger$ & $6.4(1-12)$ & $\begin{array}{l}\text { Reduction: halter traction, } \\
\text { average } 3 \text { days, } n=16 \text {; } \\
\text { retention: } N A\end{array}$ & NA & NA & $\begin{array}{l}\text { Failure to reduce, } n=4 \text {; required fusion, } \\
n=2 \text {, or further halter traction, } n=2 \text {; } \\
\text { among cases } w / \text { failure, } n=2 \text { had symp- } \\
\text { toms for }>3 \text { mos }\end{array}$ \\
\hline
\end{tabular}

* Thirty-five total cases: 29 AARF and 6 "diagnostic gray zone."

† Twenty-one cases total, with 13 having radiological AARF. 
method of retention can be divided into cervical collars and braces. Cervical collars can be further subdivided into 1) soft or Schanz collar; 2) hard collar, Philadelphia, or Miami J collar; and 3) rigid cervical orthosis or sternal occipital mandibular immobilizer (SOMI). Similarly, braces can be subdivided into 1) halo jacket, 2) Minerva jacket, and 3) Guilford brace or cervicothoracic orthosis. The use of cervical collars has been described more frequently than the use of braces (25/37 [68\%] vs $17 / 37$ [46\%]). The heterogeneity in the combination of reduction method plus retention method both among and within studies and the lack of a rationale for choosing one method over another have limited the outcome comparison between different conservative treatment methods in AARF. Further study directly comparing different methods at each stage is therefore required.

\section{Effect of Symptom Duration Before Initial Intervention on Outcomes}

There is a general consensus that early nonsurgical treatment yields a better outcome in terms of successful reduction and recurrence rates compared with those of delayed intervention. 7,16,28 The importance of early diagnosis and treatment has been highlighted by Pang and Li, who found that no acute presentations required fusion, whereas chronic cases required fusion in up to half of the cases. ${ }^{7}$ In their study, 29 patients with AARF diagnosed on the basis of clinical and radiological criteria were subdivided into acute $(\mathrm{n}=12)$, subacute $(\mathrm{n}=7)$, and chronic $(\mathrm{n}=10)$ cases according to a pretreatment delay of $<1,1-3$, and $>3$ months, respectively. Acute and subacute patients received halter traction followed by a Guilford brace, and chronic cases received skull traction followed by a halo vest. All acute cases were successfully reduced $(n=12$ [100\%]), and fusion surgery was needed in 6 patients $(35 \%)$ from the subacute and chronic groups. However, the study applied a different treatment modality between the two patient groups, with halter traction and a Guilford brace in the acute group and skull traction and a halo vest in the chronic group. ? Phillips and Hensinger reported on 23 patients and retrospectively compared success rates between those with an AARF duration $<1$ week $(n=11)$, from 1 week to 1 month $(\mathrm{n}=5)$, and $>1$ month $(\mathrm{n}=7)$ before treatment. ${ }^{48}$ All presenting $<1$ week and 1 week to 1 month had successful reductions with no recurrence. Reduction was achieved in $86 \%(n=6)$ of patients presenting after 1 month; $67 \%(n=4)$ of these patients experienced recurrences, and $33 \%(n=2)$ eventually underwent arthrodesis of the first and second vertebrae. In a retrospective study, Glotzbecker et al. compared duration of symptoms before intervention in those successfully versus unsuccessfully conservatively treated among the patients with a symptom duration over 4 weeks $(n=11){ }^{28}$ The authors concluded that in those with a successful reduction, the average duration of symptoms was 6 weeks $(n=8[73 \%])$ as compared to 9.3 weeks $(n=3[27 \%])$ in those in whom treatment had failed. Mihara et al. reported on 35 patients and compared the rate of recurrence in those with different pretreatment symptom durations. ${ }^{16}$ These authors found that all patients presenting with more than a 1-week delay $(\mathrm{n}=$ 4) had a $100 \%$ rate of recurrence. Prompt recognition and
TABLE 3. Method of reduction and retention described by studies in this systematic review

\begin{tabular}{|c|c|c|}
\hline $\begin{array}{l}\text { Initial Mode of } \\
\text { Reduction }\end{array}$ & $\begin{array}{l}\text { Mode of } \\
\text { Retention }\end{array}$ & Reference \\
\hline No intervention & No intervention & 24,43 \\
\hline \multirow{4}{*}{$\begin{array}{l}\text { No reduction/spon- } \\
\text { taneous reduction } \\
\text { w/o manipulation, or } \\
\text { traction/reduction not } \\
\text { described }\end{array}$} & Cervical collar (unspecified) & $13,22,24,44$ \\
\hline & Soft or Schanz collar & $26,43,50$ \\
\hline & $\begin{array}{l}\text { Hard, Philadelphia, or Miami } \\
\text { J collar }\end{array}$ & $\begin{array}{c}26,38,40 \\
41,43,50\end{array}$ \\
\hline & $\begin{array}{l}\text { Cervicothoracic orthosis or } \\
\text { Guilford brace }\end{array}$ & 44 \\
\hline $\begin{array}{l}\text { Anti-inflammatories } \\
\& / \text { r muscle relaxants } \\
\text { \&/or analgesia \&/or } \\
\text { physiotherapy only }\end{array}$ & Rigid cervical orthosis or SOMI & 41,43 \\
\hline \multirow{6}{*}{$\begin{array}{l}\text { Manual reduction } \\
\text { under anesthesia }\end{array}$} & Cervical collar (unspecified) & 47 \\
\hline & Soft or Schanz collar & 23 \\
\hline & $\begin{array}{l}\text { Hard, Philadelphia, or Miami } \\
\text { J collar }\end{array}$ & $23,36,45$ \\
\hline & Rigid cervical orthosis or SOMI & 27 \\
\hline & Halo jacket & 34,35 \\
\hline & Minerva jacket & 6 \\
\hline \multirow[t]{5}{*}{ Traction (unspecified) } & Cervical collar (unspecified) & 40,47 \\
\hline & Soft or Schanz collar & 37 \\
\hline & $\begin{array}{l}\text { Hard, Philadelphia, or Miami } \\
\text { J collar }\end{array}$ & 38 \\
\hline & Halo jacket & 21 \\
\hline & Minerva jacket & 30 \\
\hline \multirow[t]{7}{*}{ Halter traction } & Cervical collar (unspecified) & 30,46 \\
\hline & Soft or Schanz collar & $16,48,49$ \\
\hline & $\begin{array}{l}\text { Hard, Philadelphia, or Miami } \\
\text { J collar }\end{array}$ & 48 \\
\hline & Halo jacket & $28,45,48$ \\
\hline & Minerva jacket & 48 \\
\hline & $\begin{array}{l}\text { Cervicothoracic orthosis or } \\
\text { Guilford brace }\end{array}$ & 7 \\
\hline & Not described & 43,51 \\
\hline \multirow{5}{*}{$\begin{array}{l}\text { Halo traction/Gard- } \\
\text { ner-Wells traction }\end{array}$} & Cervical collar (unspecified) & 24,46 \\
\hline & Rigid cervical orthosis or SOMI & 22 \\
\hline & Halo jacket & $7,18,28$ \\
\hline & Minerva jacket & 18 \\
\hline & Not described & $40,43,48$ \\
\hline \multirow{2}{*}{$\begin{array}{l}\text { Novel or custom- } \\
\text { made therapy }\end{array}$} & Philadelphia collar & 33 \\
\hline & Halo vest & 32 \\
\hline
\end{tabular}

diagnosis of AARF is therefore critical, and neurosurgical consultation for all children presenting with torticollis has been recommended..$^{30}$

\section{Duration of Postreduction Position Maintenance}

The duration of treatment varied among the studies. While traction therapies were performed until successful reduction was achieved or deemed a failure, there was 
no consistency as to how long reduction therapy should be tried before considering alternatives. The longest described therapy was halo traction for up to 3 months. There was also no consensus on the duration of the stabilization phase in which patients were to remain in their collars or vests both within and among studies. The average duration ranged from 1 week to 3 months with variable outcomes. An optimal duration of conservative therapy and the effect of a prolonged duration of therapy in children still need to be addressed.

\section{Predicting AARF Recurrence}

Accurate risk stratification of AARF recurrence would allow tailored management. A total of 6 papers described the duration of subluxation before initial treatment as a

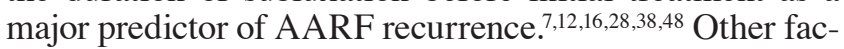
tors considered as potential risk factors in the literature included age group, etiology, and severity of AARF based on radiological and clinical presentation. While no significant difference in the recurrence rate between different age groups or etiologies of AARF has been found in comparative studies, ${ }^{12,16}$ Pang and Li reported that a greater severity of AARF (as designated by the degree of motion abnormality of the $\mathrm{C} 1-2$ complex) predisposes to its recurrence. ${ }^{7}$

\section{Optimal Second-Line Treatment Strategy}

Considering the identified studies, we were unable to define a clear time frame in which clinicians should think about escalating from first- to second-line therapy. ${ }^{11,12}$ It was not evident which treatment was optimal after the failure of nonsurgical treatment, for example, whether further nonsurgical treatment should be tried with the same or a different method and its duration or whether surgery should be performed. While Pang and Li described a second attempt at nonsurgical management with successful outcomes, ${ }^{12}$ Phillips and Hensinger recommended arthrodesis following an initial failure to reduce with cervical traction. ${ }^{48}$ This finding further highlighted the need for clear evidence on which to base management decisions in AARF, including when to escalate to surgery.

\section{General Limitations of the Included Studies}

The field of AARF and the studies in the systematic review are vulnerable to a number of general limitations outlined below.

\section{Lack of Prospective Trials and Comparative Analysis}

No studies in this review prospectively compared two different modes of nonsurgical treatment for matched patient cohorts. The only studies providing direct comparative analysis of two or more nonsurgical treatments were retrospective studies by Phillips and Hensinger, Mihara et al., Ishii et al., and Glotzbecker et al. and prospective studies by Pang and Li. 7.12,16,28,38,48 Retrospective studies are prone to recall bias and present a lower level of evidence compared with that in prospective studies. Four of these studies compared the outcomes between patient groups with different durations of symptoms before diagnosis. ${ }^{7,16,38,48}$ Additionally, Ishii et al. compared CT findings between acute and chronic AARF, and Pang and Li com- pared outcomes between different radiological subtypes of AARF. ${ }^{7,38}$ Direct comparative analysis of management types was hampered by a lack of standardization and consistency between studies. There was wide variety in patient variables (age, etiology of AARF, time to presentation or treatment, etc.) and how treatment methods were applied (including duration of therapy). In addition, 19 of the 33 retrospective studies were small case series with fewer than 10 patients.

\section{Outcome Measures}

There was widespread discrepancy in the reported primary outcome data. Many of the included papers had favorable outcomes as defined by the studies themselves, although outcome analysis is hindered by the presence of information bias within the retrospective case reviews and prospective studies. Most studies reported only selected aspects of patient outcomes with limited details, for example, rate of recurrence or imaging-based analysis but not quality of life. The psychological impacts of long-term traction and retention in young children were also not reported in the studies.

\section{Suggestions for Future Studies}

There is a pressing need for future well-designed comparative studies to investigate patient outcomes among different methods of nonsurgical treatment. Ideally, future studies would prospectively randomize AARF patients into different nonsurgical treatment arms comparing different methods of reduction and retention. If a randomization design is not possible, then patients should be matched for age or causation and duration of AARF. Outcome measures should not only include success in terms of reduction and recurrence rates, but also address pain scores and quality of life. Quality-of-life measures in the pediatric population are challenging and limited by the child's age and cognitive development. ${ }^{54}$ There are no validated disease-specific questionnaires for AARF at present; therefore, only generic quality-of-life questionnaires can be used. In addition, AARF can affect children from a very young age through to adulthood; thus, it may be appropriate for the chosen questionnaire to be targeted at parents, so that responses are valid across a varied age range. The Warwick Child Health and Morbidity Profile and the 28-item Child Health Questionnaire (CHQ-PF28) are two examples of quality-of-life outcome measures that also include pain and disability outcomes and may be suitable for AARF studies. ${ }^{55,56}$

\section{Conclusions}

There is no level I evidence comparing different nonsurgical management approaches for AARF. Most of the data included in this systematic review came from uncontrolled, nonrandomized case series. While there are a wide variety of management approaches, there seems to be a consensus that the longer the time to presentation, the higher the risk of treatment failure. Generally good outcomes are defined by the studies regardless of treatment methods. However, most primary outcome data focused mainly on radiological outcome, rate of successful reduc- 
tion, and/or rate of recurrence, with limited comment on quality of life and psychological impact of treatment on young children. Studies were limited by apparent bias in outcome reporting; management; lack of matching, randomization, or blinding; and numerous confounding factors. Therefore, further research is required to determine the optimal treatment approach for AARF.

\section{Appendix}

\section{Systematic Review Search Strategy}

The following search terms were used in this systematic review: "Atlantoaxial rotatory fixation, Atlantoaxial rotary fixation, Atlanto-axial rotatory fixation, Atlanto-axial rotary fixation, Atlantoaxial rotatory subluxation, AARF, Atlantoaxial rotary subluxation, Atlanto-axial rotatory subluxation, Atlanto-axial rotary subluxation, AARS, Atlantoaxial rotatory displacement, Atlantoaxial rotary displacement, Atlanto-axial rotatory displacement, Atlanto-axial rotary displacement, AARD, Cock robin, Cock-robin, Cock-Robin, Childhood torticollis, Paediatric torticollis, Pediatric torticollis" AND "Management, Treatment, Surgery, Surgical, Fixation, Fusion, Surgical fixation, Surgical fusion, Conservative, Conservative management, Conservative treatment, Traction, Collar, Halo."

\section{References}

1. Pang D, Li V. Atlantoaxial rotatory fixation: part 1-biomechanics of normal rotation at the atlantoaxial joint in children. Neurosurgery. 2004;55(3):614-626.

2. Jeon SW, Jeong JH, Moon SM, Choi SK. Atlantoaxial rotatory fixation in adults patient. J Korean Neurosurg Soc. 2009; 45(4):246-248.

3. Rahimizadeh A, Williamson W, Rahimizadeh S. Traumatic chronic irreducible atlantoaxial rotatory fixation in adults: review of the literature, with two new examples. Int J Spine Surg. 2019;13(4):350-360.

4. Fielding JW, Hawkins RJ. Atlanto-axial rotatory fixation. (Fixed rotatory subluxation of the atlanto-axial joint). J Bone Joint Surg Am. 1977;59(1):37-44.

5. Muñiz AE, Belfer RA. Atlantoaxial rotary subluxation in children. Pediatr Emerg Care. 1999;15(1):25-29.

6. Schwarz N. The fate of missed atlanto-axial rotatory subluxation in children. Arch Orthop Trauma Surg. 1998;117(4-5): 288-289.

7. Pang D, Li V. Atlantoaxial rotatory fixation: part 3-a prospective study of the clinical manifestation, diagnosis, management, and outcome of children with alantoaxial rotatory fixation. Neurosurgery. 2005;57(5):954-972.

8. James G, Thompson DNP. Atlanto-axial rotatory fixation: a serious potential complication of paediatric ENT surgery that requires prompt diagnosis and treatment. J Laryngol Otol. 2017;131(11):940-945.

9. Neal KM, Mohamed AS. Atlantoaxial rotatory subluxation in children. J Am Acad Orthop Surg. 2015;23(6):382-392.

10. Kinon MD, Nasser R, Nakhla J, et al. Atlantoaxial rotatory subluxation: a review for the pediatric emergency physician. Pediatr Emerg Care. 2016;32(10):710-716.

11. Crossman JE, David K, Hayward R, Crockard HA. Open reduction of pediatric atlantoaxial rotatory fixation: long-term outcome study with functional measurements. J Neurosurg. 2004;100(3)(Suppl Spine):235-240.

12. Pang D. Atlantoaxial rotatory fixation. Neurosurgery. 2010; 66(3)(suppl):161-183.

13. Beier AD, Vachhrajani S, Bayerl SH, et al. Rotatory subluxation: experience from the Hospital for Sick Children. J Neurosurg Pediatr. 2012;9(2):144-148.

14. Wang S, Yan M, Passias PG, Wang C. Atlantoaxial rotatory fixed dislocation: report on a series of 32 pediatric cases. Spine (Phila Pa 1976). 2016;41(12):E725-E732.

15. Matsuyama Y, Ishikawa T, Ozone E, et al. Fusion surgery required for recurrent pediatric atlantoaxial rotatory fixation after failure of temporary fixation with instrumentation. Case Rep Orthop. 2017;2017:1017307.

16. Mihara H, Onari K, Hachiya M, et al. Follow-up study of conservative treatment for atlantoaxial rotatory displacement. J Spinal Disord. 2001;14(6):494-499.

17. Park SW, Cho KH, Shin YS, et al. Successful reduction for a pediatric chronic atlantoaxial rotatory fixation (Grisel syndrome) with long-term halter traction: case report. Spine (Phila Pa 1976). 2005;30(15):E444-E449.

18. Mifsud M, Abela M, Wilson NI. The delayed presentation of atlantoaxial rotatory fixation in children: a review of the management. Bone Joint J. 2016;98-B(5):715-720.

19. Moher D, Liberati A, Tetzlaff J, Altman DG. Preferred reporting items for systematic reviews and meta-analyses: the PRISMA statement. Ann Intern Med. 2009;151(4):264-269, W64.

20. Tricco AC, Lillie E, Zarin W, et al. PRISMA extension for scoping reviews (PRISMA-ScR): checklist and explanation. Ann Intern Med. 2018;169(7):467-473.

21. Patel V, Yang R, Shakir S, et al. Atlantoaxial rotatory subluxation: a rare complication of craniofacial surgery. J Craniofac Surg. 2020;31(1):166-168.

22. Iaccarino C, Francesca O, Piero S, et al. Grisel's syndrome: non-traumatic atlantoaxial rotatory subluxation-report of five cases and review of the literature. Acta Neurochir Suppl. 2019;125:279-288.

23. Ozalp H, Hamzaoglu V, Avci E, et al. Early diagnosis of Grisel's syndrome in children with favorable outcome. Childs Nerv Syst. 2019;35(1):113-118.

24. Powell EC, Leonard JR, Olsen CS, et al. Atlantoaxial rotatory subluxation in children. Pediatr Emerg Care. 2017;33(2): $86-91$.

25. Ocak U, Ocak PE. A diagnose not to miss: rotatory atlantoaxial subluxation in emergency service. Cumhuriyet Med J. 2016;38(1):46-52.

26. Missori P, Marruzzo D, Peschillo S, Domenicucci M. Clinical remarks on acute post-traumatic atlanto-axial rotatory subluxation in pediatric-aged patients. World Neurosurg. 2014;82(5):e645-e648.

27. Akbay A, Bilginer B, Akalan N. Closed manual reduction maneuver of atlantoaxial rotatory dislocation in pediatric age. Childs Nerv Syst. 2014;30(6):1083-1089.

28. Glotzbecker MP, Wasser AM, Hresko MT, et al. Efficacy of nonfusion treatment for subacute and chronic atlanto-axial rotatory fixation in children. J Pediatr Orthop. 2014;34(5): 490-495.

29. Tauchi R, Imagama S, Ito Z, et al. Surgical treatment for chronic atlantoaxial rotatory fixation in children. J Pediatr Orthop B. 2013;22(5):404-408.

30. Ortiz GL, Pratts I, Ramos E. Grisel's syndrome: an unusual cause of torticollis. J Pediatr Rehabil Med. 2013;6(3):175180.

31. Dahdaleh NS, Dlouhy BJ, Menezes AH. One-step fixation of atlantoaxial rotatory subluxation: technical note and report of three cases. World Neurosurg. 2013;80(6):e391-e395.

32. Ishii K, Toyama Y, Nakamura M, et al. Management of chronic atlantoaxial rotatory fixation. Spine (Phila Pa 1976). 2012;37(5):E278-E285.

33. Ciftdemir M, Copuroğlu C, Ozcan M, et al. Non-operative treatment in children and adolescents with atlantoaxial rotatory subluxation. Balkan Med J. 2012;29(3):277-280.

34. Tauchi R, Imagama S, Kanemura T, et al. The treatment of refractory atlanto-axial rotatory fixation using a halo vest: results of a case series involving seven children. J Bone Joint Surg Br. 2011;93(8):1084-1087. 
35. Ishii K, Matsumoto M, Momoshima S, et al. Remodeling of $\mathrm{C} 2$ facet deformity prevents recurrent subluxation in patients with chronic atlantoaxial rotatory fixation: a novel strategy for treatment of chronic atlantoaxial rotatory fixation. Spine (Phila Pa 1976). 2011;36(4):E256-E262.

36. Kim B, Iwata K, Sugimoto K, et al. Significance of prevention and early treatment of a postoperative twisted neck: atlantoaxial rotatory subluxation after head and neck surgery. J Anesth. 2010;24(4):598-602.

37. Wang YF, Mu-Huo Teng M, Sun YC, et al. Torticollis due to atlantoaxial rotatory fixation. J Clin Neurosci. 2008;15(3): 316-318.

38. Ishii $\mathrm{K}$, Chiba $\mathrm{K}$, Maruiwa $\mathrm{H}$, et al. Pathognomonic radiological signs for predicting prognosis in patients with chronic atlantoaxial rotatory fixation. J Neurosurg Spine. 2006;5(5): 385-391.

39. Chiapparini L, Zorzi G, De Simone T, et al. Persistent fixed torticollis due to atlanto-axial rotatory fixation: report of 4 pediatric cases. Neuropediatrics. 2005;36(1):45-49.

40. Rahimi SY, Stevens EA, Yeh DJ, et al. Treatment of atlantoaxial instability in pediatric patients. Neurosurg Focus. 2003; 15(6):1-4.

41. Fernández Cornejo VJ, Martínez-Lage JF, Piqueras C, et al. Inflammatory atlanto-axial subluxation (Grisel's syndrome) in children: clinical diagnosis and management. Childs Nerv Syst. 2003;19(5-6):342-347.

42. Lee SC, Lui TN, Lee ST. Atlantoaxial rotatory subluxation in skeletally immature patients. Br J Neurosurg. 2002;16(2): 154-157.

43. McGuire KJ, Silber J, Flynn JM, et al. Torticollis in children: can dynamic computed tomography help determine severity and treatment. J Pediatr Orthop. 2002;22(6):766-770.

44. Martinez-Lage JF, Martinez Perez M, Fernandez Cornejo V, Poza M. Atlanto-axial rotatory subluxation in children: early management. Acta Neurochir (Wien). 2001;143(12): 1223-1228.

45. Holcomb JD, Jaffe DM, Greinwald JH Jr, et al. Nontraumatic atlantoaxial rotary subluxation in the pediatric otolaryngology patient. A report of four cases. Ann Otol Rhinol Laryngol. 2001;110(12):1137-1140.

46. Herzka A, Sponseller PD, Pyeritz RE. Atlantoaxial rotatory subluxation in patients with Marfan syndrome. A report of three cases. Spine (Phila Pa 1976). 2000;25(4):524-526.

47. Grøgaard B, Dullerud R, Magnaes B. Acute torticollis in children due to atlanto-axial rotary fixation. Arch Orthop Trauma Surg. 1993;112(4):185-188.

48. Phillips WA, Hensinger RN. The management of rotatory atlanto-axial subluxation in children. J Bone Joint Surg Am. 1989;71(5):664-668.

49. Johnson DP, Fergusson CM. Early diagnosis of atlanto-axial rotatory fixation. J Bone Joint Surg Br. 1986;68(5):698-701.
50. Landi A, Pietrantonio A, Marotta N, et al. Atlantoaxial rotatory dislocation (AARD) in pediatric age: MRI study on conservative treatment with Philadelphia collar-experience of nine consecutive cases. Eur Spine J. 2012;21(1)(suppl 1): S94-S99.

51. Nicholson P, Higgins T, Forgarty E, et al. Three-dimensional spiral CT scanning in children with acute torticollis. Int Orthop. 1999;23(1):47-50.

52. Pang $\mathrm{D}, \mathrm{Li} \mathrm{V}$. Atlantoaxial rotatory fixation: part 2-new diagnostic paradigm and a new classification based on motion analysis using computed tomographic imaging. Neurosurgery. 2005;57(5):941-953.

53. Verhofste BP, Glotzbecker MP, Birch CM, et al. Halo-gravity traction for the treatment of pediatric cervical spine disorders. J Neurosurg Pediatr. 2019;25(4):384-393.

54. Eiser C, Morse R. A review of measures of quality of life for children with chronic illness. Arch Dis Child. 2001;84(3): 205-211.

55. Spencer NJ, Coe C. The development and validation of a measure of parent-reported child health and morbidity: the Warwick Child Health and Morbidity Profile. Child Care Health Dev. 1996;22(6):367-379.

56. Raat H, Botterweck AM, Landgraf JM, et al. Reliability and validity of the short form of the child health questionnaire for parents (CHQ-PF28) in large random school based and general population samples. J Epidemiol Community Health. 2005;59(1):75-82.

\section{Disclosures}

The authors report no conflict of interest concerning the materials or methods used in this study or the findings specified in this paper.

\section{Author Contributions}

Conception and design: Hill, Borg. Acquisition of data: Hill, Sae-Huang. Analysis and interpretation of data: Hill, Sae-Huang. Drafting the article: Hill, Sae-Huang. Critically revising the article: Hill, Borg. Reviewed submitted version of manuscript: Hill. Approved the final version of the manuscript on behalf of all authors: Hill. Statistical analysis: Hill. Administrative/technical/ material support: Sae-Huang. Study supervision: Hill, Borg.

\section{Correspondence}

Ciaran Scott Hill: The National Hospital for Neurology and Neurosurgery, London, UK. ciaran.hill@ucl.ac.uk. 